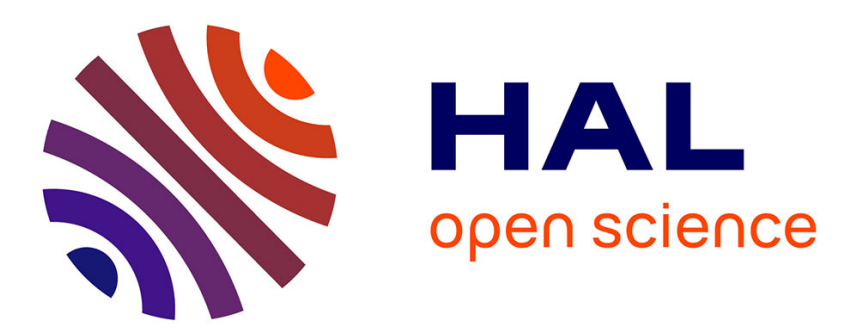

\title{
Contribuer à la diffusion du patrimoine documentaire sur Wikipédia : pratiques et enjeux pour les institutions culturelles
}

Jessica de Bideran, Romain Wenz

\section{- To cite this version:}

Jessica de Bideran, Romain Wenz. Contribuer à la diffusion du patrimoine documentaire sur Wikipédia : pratiques et enjeux pour les institutions culturelles. Culture et Musées, 2020, Musées et mondes numériques, 35, pp.165-188. 10.4000/culturemusees.4762 . hal-03242459

\section{HAL Id: hal-03242459 \\ https://hal.science/hal-03242459}

Submitted on 31 May 2021

HAL is a multi-disciplinary open access archive for the deposit and dissemination of scientific research documents, whether they are published or not. The documents may come from teaching and research institutions in France or abroad, or from public or private research centers.
L'archive ouverte pluridisciplinaire HAL, est destinée au dépôt et à la diffusion de documents scientifiques de niveau recherche, publiés ou non, émanant des établissements d'enseignement et de recherche français ou étrangers, des laboratoires publics ou privés. 


\section{Culture \& Musées}

Muséologie et recherches sur la culture

$35 \mid 2020$

Musées et mondes numériques

Dossier

\section{Contribuer à la diffusion du patrimoine documentaire sur Wikipédia : pratiques et enjeux pour les institutions culturelles}

Contributing to the circulation of documentary heritage on Wikipedia: practices and issues for cultural institutions

Contribuir con la difusión del patrimonio documental en Wikipedia: prácticas y desafíos para las instituciones culturales

\section{JESSICA DE BIDERAN ET ROMAIN WENZ}

p. $165-188$

https://doi.org/10.4000/culturemusees.4762

\section{Résumés}

Français English Español

Pour les institutions patrimoniales, la participation à des initiatives ouvertes telles que Wikipédia est un changement de paradigme. Sur la base d'une expérience d'enseignement de plusieurs années en lien avec des structures culturelles conservant du patrimoine documentaire, l'article permet de confronter les réflexions théoriques contemporaines sur la dissémination des connaissances à la réalité des institutions administratives. L'enjeu pour les acteurs est de concilier les besoins et principes d'une encyclopédie généraliste et mondiale avec les attentes d'institutions historiquement centrées sur la présentation de collections à des visiteurs physiques. Toutefois, vouloir être présent sur Wikipédia implique pour l'institution culturelle de repenser sa posture vis-à-vis de publics virtuels et non moins collectivement organisés et engagés.

Participation in open access initiatives such as Wikipedia is a shift in paradigm for heritage institutions. On the basis of several years of experience teaching in relation with cultural institutions with documentary holdings, this article proposes a comparison between current theories on the transmission of knowledge and the reality of administrative institutions. For those with a stake in such institutions, the issue is the reconciliation of the needs and principals of a general and universal encyclopedia with the expectations of institutions historically focused on the presentation of their collections to physical visitors. A cultural institution's desire to be present on Wikipedia, however, implies a rethinking of its posture with regards to virtual, and no less organized or engaged, visitors.

Para las instituciones patrimoniales, la participación en iniciativas abiertas como Wikipedia significa un cambio de paradigma. Sobre la base de una experiencia de enseñanza de varios años vinculada con estructuras culturales de conservación del patrimonio documental, el artículo confronta las reflexiones teóricas contemporáneas sobre la difusión del conocimiento con la realidad de las instituciones administrativas. El desafío para los actores consiste en conciliar las necesidades y los principios de 
una enciclopedia generalista y mundial con las expectativas de instituciones históricamente enfocadas en la presentación de colecciones a los visitantes físicos. Pero querer estar presente en Wikipedia, implica para la institución cultural repensar su postura frente a los públicos virtuales y no menos colectivamente organizados y comprometidos.

\section{Entrées d'index}

Mots-clés : Wikipédia, numérisation, éditorialisation, compétences informationnelles, usagers

Keywords: Wikipedia, digitization, editorialization, information proficiency, users

Palabras clave: Wikipedia, digitalización, editorialización, destrezas informacionales, usuarios

Notes de la rédaction

Manuscrit reçu le 16 mai 2019

Version révisée reçue le 19 février 2020

Article accepté pour publication le 26 février 2020

\section{Texte intégral}

\section{Introduction}

Après de nombreuses années de méfiance réciproque, et à la suite de la mise en place progressive de programmes de recherche et de partenariats officiels identifiant Wikipédia comme un des acteurs les plus influents du web (Moatti, 2015), les collaborations entre des institutions patrimoniales et ses divers projets satellites (Wikimédia, Wikisource, etc.) sont aujourd'hui de plus en plus nombreux (Sajus \& Leroi, 2016). Des initiatives ont émané ces dernières années d'établissements de toutes tailles, du Musée des Augustins de Toulouse (Molinié, 2014) au Centre Pompidou (Machefert, 2014) ou aux Bibliothèques et Archives nationales du Québec (BAnQ), et concernent aussi bien des monuments historiques ${ }^{1}$ que du patrimoine immatériel ${ }^{2}$. Comme le soulignait récemment Marta Severo (2018:12), cette interaction entre une communauté d'amateurs et des institutions culturelles transforme Wikipédia en une plateforme contributive culturelle, puisque ce dispositif complexe propose un « environnement numérique qui permet à des amateurs ou, plus généralement, à des acteurs de la société civile, de contribuer à la construction de savoirs liés à des objets culturels ».

2 Diffusées par les responsables de ces actions lors de journées d'étude ou dans le cadre de bilans publiés dans des revues professionnelles (Boudreau et al., 2016), certaines de ces expériences de «documentarisation du patrimoine » (Welger-Barboza, 2001:9) ont été étudiées par différents chercheurs en sciences de l'information et de la communication. Ces travaux sur cet « objet scientifique non identifié » (Barbe et al., 2015) se concentrent sur la communauté des wikipédiens, son fonctionnement et la dimension collaborative de cette nouvelle façon d'organiser et de produire des connaissances. L'angle d'analyse retenu est celui des résistances et des collaborations qui se mettent en place entre amateurs et experts : quels savoirs diffuse ainsi le musée (Fraysse, 2015) ? Comment celui-ci s'adapte-t-il aux grands principes des cultures numériques que sont la circulation des documents et des informations ainsi que le " remix » des contenus culturels (Casemajor-Loustau, 2012) ? Autant de questions qui permettent d'interroger la place des professionnels dans ces procédures de diffusion des objets patrimoniaux et de leurs substituts numériques.

Les textes et images produits sur Wikipédia lors de ces « édit-a-thons »3 peuvent en effet être perçus comme une menace par les GLAM (galleries, libraries, archives and museums) qui peuvent notamment avoir peur de perdre le contrôle sur les utilisations commerciales des documents versés dans Wikimedia Commons (Pouchot \& Peregrina, 2015). Afin de compléter ces premières analyses, nous souhaitons ici déplacer notre regard en observant ce qui se passe lorsqu'une institution culturelle fait le choix d'utiliser Wikipédia pour diffuser ses fonds patrimoniaux en multipliant ainsi les accès numériques à ses collections en ligne diffusées initialement via sa propre bibliothèque numérique. Si du côté administratif, le développement du fonctionnement en mode projet a en effet l'avantage de permettre aux administrations la mise en place d'actions ponctuelles comme ces collaborations avec des wikipédiens construites de façon « débureaucratisée » et reprenant les codes des projets privés (Guillemot \& Jeannot, 2013), comment les principes de la plus grande encyclopédie 
du monde bousculent-ils les attentes et missions des institutions culturelles ? Quelles compétences sont mobilisées du côté de l'institution et du côté de ces publics contributeurs ? Comment, enfin, ceux-ci s'emparent-ils de ces collections patrimoniales et quels sont les impacts de ce travail d'éditorialisation sur les savoirs et objets patrimoniaux diffusés?

Pour répondre à ces questions, nous nous appuierons sur une expérience associant les équipes des fonds patrimoniaux des bibliothèques de l'Université de Bordeaux et des étudiants en première année de master " Patrimoine et musées » de l'Université Bordeaux Montaigne. Ces derniers sont ici considérés comme des publics contributeurs novices, une des catégories de publics que ce type d'action permet de rencontrer et que nous détaillerons. Cette association entre une enseignante, des wikipédiens et des structures culturelles fait suite au Wiki-Day, marathon contributif organisé dans les musées de la ville de Bordeaux en octobre 20144. Une trentaine d'articles ont été créés à cette occasion, et l'ensemble des participants souhaita prolonger cette action par des projets pédagogiques avec le Musée d'Aquitaine en 2015, le Centre Jean-Moulin en 2016 et la Commission régionale des monuments historiques en 20175. Depuis 2018, le service de coopération documentaire de l'Université de Bordeaux ${ }^{6}$, qui numérise et met en ligne à travers sa propre bibliothèque numérique les ouvrages et revues les plus précieux de sa collection, accompagne les étudiants dans un enseignement intitulé "Culture numérique ». En observant les choix effectués, les difficultés rencontrées et les objets valorisés, nous suivons à la trace les changements qui s'opèrent au sein de l'institution patrimoniale lorsque celle-ci éditorialise des œuvres anciennes sur une plateforme telle que Wikipédia. Notre participation, en tant que bibliothécaire et enseignante, servira de point d'entrée à l'analyse en nous permettant de saisir la façon dont les transitions culturelles et numériques que nous connaissons influencent le fonctionnement des institutions et la circulation des collections patrimoniales sur Internet. Les données sur lesquelles nous nous appuyons sont qualitatives : à l'observation participante est associé le suivi des traces numériques produites lors de cette expérimentation grâce, notamment, aux pages « Historique » et « Discussion » des articles modifiés qui permettent de connaître précisément les actions effectuées par les rédacteurs sur Wikipédia. Ces derniers ont également été invités à produire une synthèse personnelle des actions menées et des difficultés rencontrées dans le cadre de cet enseignement sur la page dédiée au projet pédagogique. Les verbatim qui illustrent nos propos sont extraits de ces synthèses.

5 Après avoir rappelé les origines du projet et les injonctions auxquelles il répond, nous nous intéresserons aux figures des contributeurs qui se formalisent lors de ces actions. Cette étape nous permettra d'analyser les transferts de compétences auxquels nous assistons et nous amènera ensuite à nous interroger sur le dispositif lui-même. À l'image de l'architecture physique de la bibliothèque qui modèle les pratiques de recherche et de consultation des publics (Mittler, 2014), ce nouvel espace informationnel et ces nouveaux publics bouleversent les logiques professionnelles. In fine, ce sont les représentations et circulations numériques de la collection patrimoniale que nous aborderons sous l'angle de l'éditorialisation. Les quatre dimensions du patrimoine documentaire et des institutions qui en sont les gardiennes, toutes affectées comme le rappelait récemment Dominique Boullier (2017) par le numérique, seront ainsi abordées : le stock, le lieu, les professionnels et ses usagers.

\section{Ouvrir et participer, des injonctions à la fusion des compétences}

D'abord débattue sous l'angle économique et législatif, à la suite notamment du bras de fer qui opposa en 2009 la BnF et Google (Dufrêne et al. 2013), la numérisation du patrimoine documentaire engendre des modifications juridiques, techniques et organisationnelles complexes. L'injonction au numérique vis-à-vis des structures patrimoniales (Sandri, 2016) se transforme, du côté des pouvoirs publics comme des internautes, en un désir d'ouverture des données et d'accessibilité permanente entraînant une forme de " diktat de la participation » (Severo, $2018: 111)$ pour les professionnels des bibliothèques ou des archives dont les pratiques professionnelles passent de la documentarisation à la médiation (Coste, 2017). Cela oblige les institutions culturelles à 
créer des écosystèmes de connaissances riches et intuitifs permettant de satisfaire de nombreux usagers dans une « polyphonie patrimoniale » (Both \& Garcia, 2014) complexe à saisir. Les professionnels des bibliothèques multiplient les propositions : de la rétroconversion informatique des anciens catalogues papier conservés sur support imprimé aux bibliothèques numériques, en passant par l'usage des réseaux sociaux et les données ouvertes. Ces actions de diffusion documentaire en ligne s'éloignent des pratiques traditionnelles et verticales de l'institution pour multiplier des médiations considérées comme horizontales et, a priori, caractéristiques de cette « société liquide » (Evans, 2017).

Ainsi s'établiraient des contacts avec une " communauté virtuelle » grâce à une interaction dématérialisée avec des interlocuteurs agissant à distance, parfois connus uniquement sous un pseudonyme (Perea, 2014), et non situés géographiquement. Même si la mobilisation du public et la participation en ligne restent relativement modestes en termes d'engagement, force est de constater la mise en place progressive ces dix dernières années d'une activité numérique communautaire foisonnante et autonome autour des livres imprimés (Le Béchec et al., 2018) et du patrimoine documentaire (les gallicanautes de la $\mathrm{BnF}$, les booktubeurs de Lectura Plus, etc.). Les institutions sont partagées à ce sujet : doivent-elles les encourager, les encadrer ou au contraire les ignorer ? Les expériences menées ces dernières années à Bordeaux autour de Wikipédia nous permettent d'apporter des éléments de réponse à ces questions stratégiques, en détaillant notamment les interactions qui se mettent en place entre les différents publics contributeurs et en analysant les modifications que celles-ci engendrent dans les politiques documentaires des institutions culturelles.

8 Contrairement à la conception d'une communauté de consensus systématique (Langlais, 2014), la communauté contributive wikipédienne s'appuie sur l'implication de personnes intervenant physiquement et de vive voix pour former, accompagner et permettre aux nouveaux auteurs de contribuer efficacement à l'encyclopédie. Réciproquement, dans le secteur du patrimoine culturel, l'interaction entre personnes physiques permet de dépasser les postures institutionnelles en facilitant l'accès aux équipes de conservation. Ce scénario est particulièrement bien décrit par Marta Severo (2018 : 95), qui souligne que les institutions culturelles mandatent " des passionnés débutants et des éditeurs experts qui agissent dans le cadre du système d'auto-organisation de Wikipédia ». Tel est le cas dans l'expérience qui nous intéresse ici.

9 Dès l'origine du projet, bibliothécaires, enseignante et wikipédiens de l'association locale se sont réunis pour définir le périmètre du travail à effectuer. C'est au cours de ces temps d'échange (deux réunions entre juillet et septembre 2018) que les postures de chacun face aux futurs publics se sont progressivement dessinées : aux bibliothécaires le rôle de transmetteurs (d'information, de sources et de documents), à l'enseignante celui de passeur7 (de logiques éditoriales et de dynamique de travail), et aux wikipédiens confirmés ceux de médiateurs (avec la communauté de wikipédiens distante, mais vigilante à toute nouvelle modification). C'est aussi lors de ces temps d'échange que nous avons pu sélectionner les fonds et les sujets susceptibles de répondre à l'admissibilité des articles envisagés. Certaines créations n'allaient pas de soi, telle la page sur les fonds patrimoniaux de la bibliothèque universitaire dont l'existence a été soumise à discussion ${ }^{8}$ avec des wikipédiens ( $c f$. infra).

Comme le souligne Patrick Fraysse (2015) au sujet des ateliers Wikimédia menés au Musée des Augustins de Toulouse, le premier transfert de compétences qui apparaît lors de ce type d'action est celui des professionnels du patrimoine vers les wikipédiens : « il ne s'agit plus de faire, mais de faire faire en supervisant les opérations ». Ces apports relèvent des compétences des bibliothécaires qui vont être en mesure soit de délivrer des connaissances historiques ou bibliothéconomiques sur tels ouvrages ou auteurs, soit d'identifier les objets patrimoniaux les plus pertinents pour illustrer tel article. Mais d'autres compétences entrent aussi en jeu, en particulier celles des wikipédiens confirmés qui, par leur statut de contributeurs réguliers connaissant les grands principes éditoriaux de l'encyclopédie, assurent un rôle de médiateurs entre l'institution et la communauté de wikipédiens qui restent derrière leurs écrans et surveillent les actions des nouveaux entrants. Evelyne Broudoux (2015) a récemment rappelé que Wikipédia " ne dévoile pas facilement ses règles ", avec l'internalisation de l'organisation et des règles d'écriture, ce fonctionnement ne facilitant pas finalement l'arrivée de nouveaux entrants s'ils ne sont pas accompagnés de contributeurs reconnus 9 .

Alors que l'injonction à participer, caractéristique du web des plateformes (Proulx, 2017), entraine du côté des professionnels des questions sur l'" efficacité documentaire » (Casemajor-Lousteau, 2011) de ces contributions, cette expérience permet de constater que 
les rôles de producteur de données patrimoniales (les documents numériques, leurs métadonnées, les informations associées, etc.) et d'usagers de ces données (les wikipédiens et les publics contributeurs) fusionnent dans une nouvelle figure de « professionnel amateur » où chacun s'enrichit des compétences et connaissances des uns et des autres. Cette implication participe d'une sorte de reconnaissance, par la communauté, mais aussi et surtout par l'institution, de la valeur sociale de ces sujets médiateurs externes à l'institution (Honneth, 2004).

Notre expérience autorise à relativiser les craintes de désintermédiation et de dématérialisation souvent associées à l'introduction du numérique dans les structures culturelles. Au contraire, dans ce type de projets " l'humain se trouve au centre de tout » (Michel et al., 2013) et cela entraîne progressivement des transferts de compétences non pas seulement de l'institution vers ces publics usagers et contributeurs, mais aussi de ces derniers vers l'institution. Construire un tel projet suppose aussi d'agir en toute transparence pour expliciter la collaboration en cours et ouvrir celle-ci à toute la communauté des wikipédiens, ceux impliqués physiquement in situ, mais aussi ceux en ligne qui réagiront aux modifications et publications. Cette transparence est assurée par l'existence d'une page du projet pédagogique qui nous permet de cadrer le travail des différents publics par des consignes et des échéances, mais aussi de recueillir leurs ressentis et alerter la communauté des wikipédiens sur le caractère novice de ces contributeurs. Car les « patrouilleurs $»^{10}$ sont particulièrement redoutés par les novices, comme le souligne ce témoignage : « La communauté wikipédienne nous a supprimé une première fois notre article [...] » (L. et J.). Et si, par cette dimension humaine et sociale, l'on peut rapprocher Wikipédia d'un « service de réseautage social comme les autres » (Cardon \& Casilli, 2015), il convient aussi de s'interroger sur la façon dont cette plateforme numérique façonne ces nouvelles pratiques.

\section{Sélectionner et collaborer, éclatement des publics et des usages de la collection}

Pour pallier les écueils de Wikipédia, encyclopédie rattachée à une culture geek (Cardon 2016) et centrée sur un Internet très masculin (Dagnaud 2018), des établissements culturels français ont conventionné à partir des années 2010 avec l'association Wikimédia France afin de mettre en œuvre des rendez-vous réguliers dans le but de promouvoir la représentation des institutions patrimoniales françaises dans les réseaux numériques (château de Versailles en 2011, Musée du Quai-Branly en 2013, Musée des arts décoratifs de Paris en 2014, etc.).

Car Wikipédia offre en effet un " cadre frontière " (Flichy, 2003) qui repose sur un ensemble d'artefacts techniques (le logiciel MediaWiki ${ }^{11}$ principalement) et sur un ensemble de savoirs et de savoir-faire qui structurent les interactions avec l'outil et avec la communauté. Celui-ci influence le fond comme la forme des contenus. Le processus éditorial repose ainsi sur cinq grands principes ${ }^{12}$ permettant d'autoréguler les mises en ligne de nouveaux articles et la discussion entre les auteurs : (1) "Wikipédia est une encyclopédie " qui incorpore des éléments d'encyclopédie généraliste pouvant être référencés et n'accepte pas les travaux inédits ; (2) Wikipédia repose sur la " neutralité de point de vue ", autrement dit, les articles ne doivent pas promouvoir de point de vue particulier, mais rapporter des faits vérifiables ; (3) la « liberté du contenu » de Wikipédia suppose que chacun est autorisé à créer, copier, modifier et distribuer les contenus disponibles ; (4) Wikipédia suppose un " savoir-vivre communautaire » qui oblige les wikipédiens à se respecter et à dialoguer pour faire valoir leur point de vue ; (5) enfin, « Wikipédia n'a pas d'autres règles fixes ». Mélanie Larché, chargée entre 2015 et 2017 du projet collaboratif d'inventaire du patrimoine culturel immatériel (PCI) français en collaboration avec Wikipédia et le ministère de la Culture, rapporte ainsi à Marta Severo le " délicat travail de négociation entre les différents contributeurs et entre les contributeurs et les règles de la plateforme » $(2018: 103)$.

Car le premier principe, celui de l'absence de travaux inédits, est le plus complexe à saisir pour les nouveaux entrants puisqu'il suppose d'avoir connaissance à la fois des critères d'admissibilité des articles, mais aussi de référencement des sources bibliographiques. Ainsi, les wikipédiens novices expriment souvent leur incompréhension à ne pouvoir utiliser les informations informelles qu'ils recueillent lors d'échanges oraux et entretiens auprès des 
professionnels du patrimoine engagés dans ces projets. Surtout, ce principe est souvent mal compris ; s'il interdit en effet aux chercheurs d'utiliser Wikipédia pour publier des recherches en cours, il n'interdit pas en revanche de proposer des formulations nouvelles ou, fonction intéressante pour les structures culturelles qui conservent des fonds patrimoniaux importants, d'intégrer des documents numériques nouveaux via Wikimedia Commons, la médiathèque ouverte adossée à Wikipédia. Compte tenu de ces principes, la présence sur Wikipédia repose sur deux mouvements a priori antagonistes pour une institution culturelle : d'une part le désir d'atteindre des objectifs tangibles (de rédaction d'articles, de valorisation de fonds patrimoniaux et de visibilité de l'institution), d'autre part la prise en compte d'une construction collaborative et évolutive de connaissances résultant des choix d'une communauté libre et autonome. Ainsi, l'institution est amenée à assumer le caractère incomplet du projet puisque seuls les éléments retenus par le jugement entre pairs de wikipédiens anonymes auront le droit de figurer dans l'encyclopédie. Il est dès lors inutile pour l'institution de prétendre conduire sur Wikipédia une politique documentaire au sens où les bibliothèques l'entendent traditionnellement (Mesguish, 2017).

Ici, la bibliothèque se positionne en prestataire de services pour un projet ouvert qui permet de démultiplier les canaux de diffusion auprès des publics, selon sa vocation première d'ouverture et d'accessibilité. Cette posture n'est pas sans conséquence sur les missions et fonctions des professionnels de l'information puisqu'elle oblige à s'adapter à une volonté extérieure pour répondre à des demandes potentiellement tentaculaires de référencement bibliographique, de rédaction d'articles et de versement de documents. Pour l'institution patrimoniale, il s'agit d'accepter une perte d'emprise sur la propriété et les modes de production des connaissances tout en participant au renforcement d'une organisation des savoirs qui reste cohérente avec le modèle de création papier, comme l'attestent par ailleurs les diverses études qui montrent que très peu d'erreurs existent dans Wikipédia puisque la question des sources y demeure primordiale (Doutreix, 2018), perspective qui rejoint in fine les logiques bibliographiques des bibliothécaires (Sahut, 2014).

17 Dans cet éclatement des missions, les professionnels de la conservation patrimoniale posent un diagnostic sur les besoins d'information en ligne pour intervenir là où ils apporteront de la valeur ajoutée. Concrètement, une fois les trois thématiques pouvant être travaillées grâce aux fonds de la bibliothèque, identifiées et validées par les wikipédiens ${ }^{13}$, des requêtes à partir de Wikidata ont permis aux bibliothécaires d'identifier une série de pages « à l'état d'ébauche » dans la section francophone de Wikipédia. Bien que ce travail de sélection corresponde aux types de requêtes classiques mis en place par les professionnels de l'information lors des actions de numérisation pour connaître les appétences des internautes qui consultent les bibliothèques numériques, il reste cependant novateur de confronter ces données aux besoins d'un dispositif tel que Wikipédia.

Comme le souligne Olivier Ertzscheid (2008), qui insiste sur le caractère évolutif de ce projet encyclopédique, les ébauches d'articles tout comme l'ensemble des pages d'aide et des discussions sont intégralement publiques. Or, ce mode de fonctionnement ouvert, classique dans les institutions du web ( $\mathrm{W}_{3} \mathrm{C}$, par exemple), peut effrayer les administrations qui se trouvent ainsi exposées à des mises en cause parfois brutales et définitives, y compris sur des sujets relatifs à la sécurité nationale (Libération, 2013). Les institutions peuvent, dès lors, craindre de ne pas être ménagées et de devoir se justifier auprès d'une communauté qu'elle ne maîtrise pas ${ }^{14}$, ce qui fut d'ailleurs expérimenté à plusieurs reprises par les usagers novices dont la principale « difficulté fut la réception de notre article par les autres utilisateurs » (L., S., A.-S.). Les établissements culturels souhaitant publier sur Wikipédia font donc plus généralement le choix de passer par une série d'intermédiaires qui leur évitent de contribuer directement à des articles.

Habituées à des modes de négociation traditionnels, les institutions publiques doivent appréhender une règle non écrite, mais inscrite dans l'architecture même du dispositif wiki et qui peut être dissuasive : la publication en ligne de toutes les discussions sans limitation de durée. L'analyse de ces échanges avec la communauté donne à voir un éclatement des profils d'usagers « virtuels » interagissant à distance avec les usagers " physiques » qui travaillent à partir des collections (bibliothécaires, enseignants, étudiants). Nous pouvons en distinguer au moins quatre : les « pompiers » insistent sur la nomenclature, le découpage des textes et la mise en forme des textes (wikification), quand les " gardiens » vivent les modifications des novices sur les pages qu'ils ont contribué à créer comme une intrusion sur leur « fief virtuel » (Chiriac, 2015) ; les " médiateurs » permettent, enfin, d'expliciter les choix et de faire le lien entre les usagers novices et les wikipédiens confirmés. À la fusion des 

contenus.

\section{Publier et éditorialiser les collections patrimoniales sur les réseaux numériques}

Depuis 2015, les actions de valorisation se sont diversifiées, passant de la modification ou création d'articles (38 en 4 ans) au versement de fac-similés numériques de qualité (plus de 150 images versées en 4 ans), en passant par l'ajout de liens depuis des pages de Wikipédia vers les bibliothèques numériques patrimoniales des universités de Bordeaux. Ce travail de publication modifie en premier lieu la place des publics qui deviennent des usagers des collections patrimoniales. La perte d'emprise sur la numérisation et surtout sur la sélection des ressources patrimoniales correspond de fait à une évolution du rôle des publics des institutions culturelles. Ainsi, le rôle d'expert et de médiateurs des wikipidiens qui nous ont accompagnés a ici été crucial, ces derniers ayant continuellement accompagné les usagers novices sur les actions possibles en les aidant à trouver leur propre place de contributeurs leur permettant d'être à l'aise et efficaces. Ils sont ainsi confrontés à une structure complexe qui les oblige à endosser des rôles complémentaires et non interchangeables; si certains s'orientent directement vers l'écriture d'articles et se révèlent parfaitement à l'aise dans ce processus créatif, d'autres sont plus à l'aise avec l'identification et la numérisation de documents illustrant des articles. Ces derniers se chargent ainsi des missions de recherche iconographique, de structuration et de versement d'images dans Wikimedia Commons, processus qui prolonge les missions classiques des bibliothécaires et documentalistes. Mais la mobilisation de leur libre arbitre, les usagers novices devant être en mesure tout au long du projet de forger leur propre opinion sur ce à quoi ressemblent de « bons » articles, les conduit progressivement à s'emparer des codes de l'encyclopédie libre, au point d'être assimilés par la communauté à de nouveaux contributeurs plus qu'à des professionnels du patrimoine qu'ils sont pourtant en devenir.

La participation des publics modifie aussi Wikipédia en enrichissant cette plateforme de contenus originaux. Il a ainsi été décidé d'identifier et de verser dans Wikimedia Commons un maximum de documents numérisés, nouveaux et disponibles sur la bibliothèque numérique patrimoniale de l'Université de Bordeaux : BabordNum ${ }^{15}$, mais pas nécessairement visibles par les wikipédiens, afin de renouveler l'approche et les discours sur les différents sujets traitables. Pour une quinzaine d'articles édités - créés ou plus souvent modifiés - dans le cadre du projet, plusieurs centaines de documents numériques ont été versés et indexés dans la médiathèque libre, telles les planches de botanique d'EdmondGustave Camus extraites d'un ouvrage de 1921, Iconographie des orchidées d'Europe et du bassin méditerranéen. Parallèlement au versement de ces planches, Wikimedia Commons a pu également être enrichi d'un ensemble de clichés présentant des signatures et des $e x$ libris aux armes d'anciens parlementaires bordelais préalablement collectés par le service du patrimoine documentaire au sein d'ouvrages conservés à la bibliothèque universitaire droit, science politique et économie. Ces fragments d'images, rares, sont principalement utiles pour les bibliophiles et ont de fait été très bien accueillis par la communauté.

Pour l'institution culturelle, cette dynamique de sélection et de republication dans la sphère wikipédienne de fragments de documents modifie, enfin, la représentation de cette collection numérique. Ce processus d'éditorialisation, défini en sciences de l'information et de la communication, dépasse en effet la simple transposition dans l'espace numérique des pratiques éditoriales du papier et participe à la production et à la structuration de l'environnement numérique grâce aux diverses interactions individuelles et collectives des publics que nous sommes tous (Vitali-Rosati, 2016). Pour les structures culturelles, "l'éditorialisation est la conclusion logique du processus de numérisation des contenus " (Bachimont, 2007). Les musées et bibliothèques patrimoniales ont ainsi repris ce terme pour nommer et définir les pratiques de médiation et de médiatisation de contenus culturels en ligne. Les dispositifs éditoriaux proposés, tels que les expositions virtuelles ou les éditions augmentées, permettent en effet de " transformer l'information en signification pour un large public » (Laborderie, 2018) et deviennent les compléments logiques des vastes bases de données qui structurent l'accès aux corpus documentaires numérisés et mis en ligne. Ces nombreuses mises en ligne, les connexions entre les différentes plateformes de 
diffusion, les interactions entre les internautes et la circulation des documents ne sont toutefois pas sans conséquence sur la perception de l'étendue des collections patrimoniales. Si l'on convient que « dans un monde numérique connecté, exister signifie être éditorialisé » (Vitali-Rosati, 2016), il faut alors reconnaître que l'éditorialisation tend à produire la réalité même.

Initier des publics à Wikipédia c'est donc aussi leur proposer une éducation critique au monde numérique en leur permettant de comprendre que ces actions contribuent à une certaine construction sociale de la réalité. Les contributeurs novices ont en effet rapidement conscience de ce morcellement des savoirs et des objets patrimoniaux dont ils s'emparent et qui permet « d'améliorer la pertinence et la visibilité de notre ajout pour le valoriser au mieux » (M. et L.) en intégrant, par exemple, des hyperliens vers d'autres pages Wikipédia. Se faisant, se présente aussi le risque d'une concentration de l'offre et de la demande sur les documents déjà numérisés. Il s'agit donc aussi de faire prendre conscience aux publics que seule une toute petite partie du patrimoine écrit est actuellement numérisée et visible sur le web. Cet aspect semble bien perçu par les contributeurs qui nous précisent ainsi avoir apprécié « aborder une nouvelle approche plus participative de Wikipédia » (A., T. et S.) tout en ayant conscience du caractère incomplet de ce travail, en indiquant, par exemple, " un axe d'amélioration [de leur travail] en allant recueillir des photographies directement sur les lieux de conservation de ces collections ce qui ne nous a pas été possible par manque de temps » (M. et L.).

L'intégration de documents numérisés et sélectionnés par les publics eux-mêmes s'inscrit dans un mouvement plus large d'évolution du web, les usagers étant aujourd'hui au centre des dispositifs et leur participation facilitée par l'aisance avec laquelle ces nouvelles plateformes peuvent aujourd'hui être prises en main. Songeons à cet égard à l'évolution de l'interface de modification des articles Wikipédia qui permet désormais de se passer de connaissance en syntaxe wiki en utilisant l'éditeur visuel dont les fonctionnalités se rapprochent des outils de bureautique et qui reste très largement exploité par les publics novices dans ce type d'actions, ces derniers ayant souvent du mal à se « familiariser avec le wikicode » (C. et E.). Ce sont ces mutations qui permettent le développement de grands collectifs en ligne ou de communautés élargies d'usagers organisés en réseaux et structurés $a$ priori de manière non hiérarchique et qui font naître des modèles stratégiques originaux tels que la numérisation à la demande.

\section{Conclusion}

Ce type d'action associant communauté virtuelle, publics et structure culturelle permet d'atteindre un objectif précis : la construction de savoirs liés à des objets patrimoniaux et la mise en circulation de documents et fac-similés numériques jusque-là consultables dans les seules bases de données patrimoniales. À cette intention première, qui peut se mesurer à l'aune du nombre d'articles édités et d'images versées, s'ajoutent aussi des résultats moins quantifiables, mais pour autant essentiels dans un contexte où citoyens comme institutions reçoivent l'injonction d'être présents et actifs sur le web. En particulier, la notoriété et l'affluence dont dispose Wikipédia permettent d'améliorer rapidement la visibilité de la structure culturelle impliquée et ainsi de valoriser ses fonds patrimoniaux auprès d'un public distant qui ne connaissait pas son existence.

De fait, l'introduction des cultures numériques dans les établissements patrimoniaux repose non seulement sur l'élaboration d'une culture technique commune, mais aussi et avant tout sur la mise en place d'une culture participative conjointe. En tant que futurs professionnels de la culture, les étudiants de niveau master sont particulièrement à même d'intervenir efficacement puisqu'ils sont à la fois sur la route de la professionnalisation et aussi indépendants que possible vis-à-vis des institutions administratives. Il s'agit ainsi autant d'« enseignement participatif » que d'« apprentissage connecté » (Jenkins et al., 2017 : 167), avec une grande importance de l'enseignement en présentiel pour transmettre et échanger en classe sur le mode de fonctionnement des communautés. L'apprentissage concerne d'ailleurs essentiellement la capacité à intégrer le mode de fonctionnement de Wikipédia et des projets satellites. C'est l'occasion ici de remercier le mentorat toujours bienveillant des wikipédiens qui nous accompagnent et transmettent avec enthousiasme leur expérience. La qualité des contenus produits par les étudiants devient en définitive 
secondaire puisqu'elle sera validée et inévitablement modifiée par la communauté, comme le montre systématiquement l'historique des articles modifiés ou créés par les étudiants.

Enfin, la numérisation de documents inédits est également l'occasion d'initier ces futurs professionnels aux enjeux juridiques qui entourent la numérisation du patrimoine et sa mise en circulation sur les réseaux numériques, sujet encore épineux pour les institutions culturelles françaises comme le rappelait récemment le programme de recherche " Images/Usages » porté par l'INHA ${ }^{16}$. Cette partie juridique serait sans doute la plus facile à transposer à d'autres institutions ou lieux, car ces enjeux réapparaissent d'une année sur l'autre, tandis que le processus d'éditorialisation est à réinventer chaque année en fonction des structures et objets patrimoniaux concernés.

Cette démarche entraîne de nouvelles manières de diffuser les objets patrimoniaux, mais aussi de nouvelles façons d'enseigner et de collaborer avec les publics : le document numérisé, " double numérique " créé à cette occasion pour illustrer des articles encyclopédiques, devient un objet patrimonial nouveau, qui est remis aux mains d'une communauté virtuelle dont le fonctionnement est, à sa manière, aussi codifié que celui des institutions culturelles.

\section{Bibliographie}

Barbe (Lionel), Merzeau (Louise) \& Schafer (Valérie) (dir.). 2015. Wikipédia, objet scientifique non identifié. Nanterre : Presses universitaires de Paris-Nanterre, en ligne : http://books.openedition.org/pupo/4079 [consulté le 6 avril 2020].

DOI : $10.4000 / 1895.2$

Bachimont (Bruno). 2007. « Nouvelles tendances applicatives : de l'indexation à l'éditorialisation », p. 15-29 in L'Indexation multimédia : Description et recherche automatiques / sous la direction de Patrick Gros. Paris : Hermès Science - Lavoisier, en ligne : http://cours.ebsi.umontreal.ca/sci6116/Ressources_files/BachimontFormatHerme\%CC\%80s.pdf [consulté le 6 avril 2020].

Both (Anne) \& Garcia (Guillaume). 2014. " Le chercheur, l'archiviste et le webmaster : la polyphonie patrimoniale? Le cas BEQuali, banque d'enquêtes qualitatives en sciences sociales », p. 353-364 in Heritage and Digital Humanities / sous la direction de Bernadette Saou-Dufrêne. Berlin : LIT.

Boudreau (Denis), Daveau (Florian) \& Giuliano (Frédéric). 2016. « Diffuser, partager et s’approprier le patrimoine documentaire québécois. Le projet collaboratif de BAnQ sur Wikimédia : une première au Canada ». Archives, 46(1), p. 61-81, en ligne : https://doi.org/10.7202/1035723ar [consulté le 6 avril 2020].

DOI : 10.7202/1035723ar

Boullier (Dominique). 2017. "Préface » in Des tweets et des likes en bibliothèque : Enquête sur la présence de quatre bibliothèques de lecture publique sur les réseaux sociaux numériques / sous la direction de Marie-Françoise Audouard, Mathilde Rimaud et Louis Wiart. Paris : Éditions de la BPI, en ligne : http://books.openedition.org/bibpompidou/2077 [consulté le 6 avril 2020].

DOI : $10.4000 / 1895.2$

Broudoux (Evelyne). 2015. "Wikipédia, objet de recherches : entre observations, expérimentations et co-constructions », p. 55-73 in Wikipédia, objet scientifique non identifié / sous la direction de Lionel Barbe, Louise Merzeau et Valérie Schafer. Nanterre : Presses universitaires de Paris-Nanterre, en ligne : http://books.openedition.org/pupo/4097 [consulté le 6 avril 2020].

DOI : $10.4000 / 1895.2$

Cardon (Dominique). 2016. «Wikipédia : comment ils ont réussi ». Sciences Humaines, 282(6), p. 2525, en ligne : https://www.cairn.info/magazine-sciences-humaines-2016-6-page-25.htm [consulté le 6 avril 2020].

Cardon (Dominique) \& Casilli (Antonio A.). 2015. Qu'est-ce que le Digital Labor ? Bry-sur-Marne : INA éditions (Études \& controverses).

Casemajor-Loustau (Nathalie). 2012. « La participation culturelle sur Internet : encadrement et appropriations transgressives du patrimoine numérisé ». Communication \& Langages, 171, p. 81-98.

Casemajor-Loustau (Nathalie). 2011. "La contribution triviale des amateurs sur le web : quelle efficacité documentaire ? ». Études de communication, 36, en ligne : http://edc.revues.org/2532 [consulté le 6 avril 2020].

DOI : $10.4000 /$ edc. 2532

Chiriac (Emanuela). 2015. « Wikipédia, la chimère du savoir libre ». Documentation et bibliothèques, 61(4), p. 159-166, en ligne : https://doi.org/10.7202/1033436ar [consulté le 6 avril 2020].

DOI : $10.7202 / 1033436$ ar

Costes (Mylène). 2017. « La valorisation du patrimoine ancien sur les sites de bibliothèques ». Bulletin des bibliothèques de France $(B B F), 12$, p. 90-103, en ligne : http://bbf.enssib.fr/consulter/bbf-201712-0090-002 [consulté le 6 avril 2020].

Dagnaud (Monique). 2018. «Internet, une passion masculine ». Le Débat, 200(3), p. 123-142, en ligne : https://www.cairn.info/revue-le-debat-2018-3-page-123.htm [consulté le 6 avril 2020]. 
DOI : 10.3917/deba.200.0123

Doutreix (Marie-Noëlle). 2018. « La fausse information au regard des vertus épistémiques de Wikipédia ». Le Temps des médias, 30, p. 91-104, en ligne : https://www.cairn.info/revue-le-tempsdes-medias-2018-1-page-91.htm [consulté le 6 avril 2020].

DOI : 10.3917/tdm.030.0091

Dufrêne (Bernadette), Ihadjadene (Madjid) \& Bruckmann (Denis). 2013. Numérisation du patrimoine : Quelles médiations ? Quels accès ? Quelles cultures ? Paris : Hermann.

Ertzscheid (Olivier). 2008. "Wikipédia est un "projet" encyclopédique et un bien commun de l'humanité ». Libération, entretien avec Astrid Girardeau, 17 janvier 2008, en ligne : http://ecrans.liberation.fr/ecrans/2008/o1/17/wikipedia-est-un-projet-encyclopedique-et-un-biencommun-de-l-humanite_954216 [consulté le 6 avril 2020].

Evans (Christophe). 2017. "Avant-propos » Des tweets et des likes en bibliothèque : Enquête sur la présence de quatre bibliothèques de lecture publique sur les réseaux sociaux numériques / sous la direction de Marie-Françoise Audouard, Mathilde Rimaud et Louis Wiart. Paris : Éditions de la BPI, en ligne : http://books.openedition.org/bibpompidou/2106 [consulté le 6 avril 2020].

DOI : $10.4000 / 1895.2$

Flichy (Patrice). 2003. L'Innovation technique. Paris : La Découverte.

Fraysse (Patrick). 2015. "La médiation numérique du patrimoine : quels savoirs au musée ? ». Distances et médiations des savoirs, [en ligne], $12:$ http://journals.openedition.org/dms/1219 [consulté le 6 avril 2020].

Guillemot (Danièle) et Jeannot (Gilles). 2013. « Modernisation et bureaucratie, l'administration d'État à l'aune du privé ". Revue française de sociologie, 54, p. 83-110, en ligne : https://www.cairn.info/revue-francaise-de-sociologie-2013-1-page-83.htm [consulté le 6 avril 2020]. DOI : $10.3917 /$ rfs.541.0083

Honneth (Axel). 2004. « La théorie de la reconnaissance : une esquisse ». Revue du MAUSS, 23, p. 133-136, en ligne : 10.3917/rdm.023.0133 [consulté le 6 avril 2020].

DOI : $10.3917 / \mathrm{rdm} .023 .0133$

Jenkins (Henry), Ito (Mizuko) \& Boyd (Danah). 2017. Culture participative. Une conversation sur la jeunesse, l'éducation et l'action dans un monde connecté. Caen : C\&F Éditions.

Laborderie (Arnaud). 2018. "Mettre en récit les données culturelles des bibliothèques numériques : exposition virtuelle et recherche-création » in Colloque international sur les bibliothèques et archives à l'ère des humanités numériques. Paris, octobre 2018, en ligne : https://hal.archives-ouvertes.fr/hal01891073 [consulté le 6 avril 2020].

Langlais Pierre-Carl, 2014. « La négociation contre la démocratie : le cas Wikipédia ». Négociations, 21, p. 21-34, en ligne : https://www.cairn.info/revue-negociations-2014-1-page-21.htm [consulté le 6 avril 2020].

DOI : 10.3917/neg.021.0021

Le Béchec (Mariannig), Boullier (Dominique) \& Crépel (Maxime). 2018. Le Livre-échange. Vies du livre \& pratiques des lecteurs. Caen : C\&F éditions (Culture numérique).

Libération. 2013. "La DCRI accusée d'avoir fait supprimer "sous la menace" un article sur Wikipédia ». Libération, 7 avril 2013, en ligne : https://www.liberation.fr/societe/2013/04/o7/ladcri-accusee-d-avoir-fait-supprimer-sous-la-menace-un-article-sur-wikipedia_894252 [consulté le 6 avril 2020].

Machefert (Sylvain). 2014. " Amélioration des articles sur l'art moderne dans Wikipédia. Partenariat entre Wikimédia France et le Centre Pompidou » in Préconférence IFLA 2014 - Bibliothèques d'art, en ligne : http://iflaparis2014.sciencesconf.org/35928 [consulté le 6 avril 2020].

Mesguich (Véronique). 2017. " Analyser et filtrer la surabondance », p. 145-160 in Bibliothèques : Le web est à vous / sous la direction de Véronique Mesguich. Paris : Éditions du Cercle de la Librairie, en ligne : https://www.cairn.info/bibliotheques-le-web-est-a-vous--9782765415213-page-145.htm [consulté le 6 avril 2020].

Michel (Jean), Mathis (Rémi), Courrier (Serge), Christophe Deschamps, Frédéric Martinet, Mathilde Bras, Jean-Baptiste Soufron, Valérie Peugeot, Anne-Marie Libmann et Véronique Mesguich. 2013. « Le contexte professionnel ». Documentaliste - Sciences de l'Information, 50, p. 48-63, en ligne : https://www.cairn.info/revue-documentaliste-sciences-de-l-information-2013-4-page-48.htm [consulté le 6 avril 2020].

Mittler (Elmar). 2014. " Les bâtiments de bibliothèques au banc d'essai : une évaluation des bâtiments des bibliothèques de recherche en Allemagne », p. 395-408 in Bibliothèques d'aujourd'hui. À la conquête de nouveaux espaces / sous la direction de Marie-Françoise Bisbrouck. Paris : Éditions du Cercle de la Librairie, en ligne : https://www.cairn.info/bibliotheques-d-aujourd-hui-9782765414292-page-395.htm [consulté le 6 avril 2020].

Moatti (Alexandre). 2015. "Postures d'opposition à Wikipédia en milieu intellectuel en France ", p. 123-133 in Wikipédia, objet scientifique non identifié / sous la direction de Lionel Barbe, Louise Merzeau et Valérie Schafer. Nanterre : Presses universitaires de Paris-Nanterre, en ligne : http://books.openedition.org/pupo/4113 [consulté le 6 avril 2020].

DOI : $10.4000 / 1895.2$

Molinié (Christelle). 2014. "Médiation documentaire et documentation participative à travers les projets Wikimédia : retour d'expérience au Musée des Augustins de Toulouse », en ligne : https://archivesic.ccsd.cnrs.fr/sic_01291772 [consulté le 6 avril 2020]. 
Perea (François). 2014. « Pseudonyme en ligne. Remarques sur la vérité et le mensonge sur soi ». Sens-Dessous, 14, p. 15-22, en ligne : https://www.cairn.info/revue-sens-dessous-2014-2-page-15.htm [consulté le 6 avril 2020].

Pouchot (Stéphanie) \& Peregina (Rafael). 2015. " GLAM et projets Wikimédia : des cordonniers mal chaussés ? ». Arbido, 15, p. 16-18.

Proulx (Serge). 2017. «L'injonction à participer au monde numérique ». Communiquer, 20, en ligne : http://journals.openedition.org/communiquer/2308 [consulté le 6 avril 2020].

DOI : $10.4000 /$ communiquer.2308

Sahut (Gilles). 2014. " "Citez vos sources" : archéologie d'une règle au cœur du savoir wikipédien (2002-2008) ». Études de communication, 42, p. 97-110, en ligne : https://www.cairn.info/revueetudes-de-communication-2014-1-page-97.htm [consulté le 6 avril 2020].

DOI : $10.4000 /$ edc.5721

Sajus (Bertrand) \& Leroi (Marie-Véronique). 2016. " Le développement du web des données culturelles. Les enjeux pour le ministère de la Culture et de la Communication ». I2D : Information, données \& documents, 53(2), p. 46-47, en ligne : https://www.cairn.info/revue-i2d-informationdonnees-et-documents-2016-2-page-46.htm [consulté le 6 avril 2020].

Sandri (Éva). 2016. L'Imaginaire des dispositifs numériques pour la médiation au musée d'ethnographie. Thèse de doctorat, Université d'Avignon.

Severo (Marta). 2018. Plateformes contributives patrimoniales. Entre institution et amateur. Mémoire d'HDR en sciences de l'information et de la communication, Université de Lille.

Vitali-Rosati (Marcello). 2016. "Qu'est-ce que l'éditorialisation ? ». Sens public, [en ligne], 18 mars 2016 : http://sens-public.org/articles/1184/ [consulté le 6 avril 2020].

Welger-Barboza (Corinne). 2001. Le Patrimoine à l'ère du document numérique. Du musée virtuel au musée médiathèque. Paris, L’Harmattan.

\section{Notes}

1 À l'image du concours international et annuel « Wiki Loves Monuments » : https://fr.wikipedia.org/wiki/Wiki_Loves_Monuments [consulté le 6 avril 2020].

2 Citons à cet égard l'usage de Wikimedia Commons par les artisans de la Manufacture nationale de Sèvres qui se servent de cette vaste médiathèque pour documenter et valoriser leurs nombreux savoirfaire en versant des images et des vidéos des étapes de production : https://commons.wikimedia.org/wiki/Category:Workshops_in_Manufacture_nationale_de_Sèvres [consulté le 6 avril 2020].

3 Nous utilisons à dessein ce mot-valise créé par la communauté des wikipédiens pour nommer ce type d'« événement organisé dans les communautés de projets en ligne tels que Wikipédia, OpenStreetMap ou encore LocalWiki pendant lequel les éditeurs créent, modifient et améliorent des articles sur un thème, sujet ou un type spécifique de contenu ». Voir : https://fr.wikipedia.org/wiki/Edit-a-thon [consulté le 6 avril 2020].

4 Voir, à cet égard : https://fr.wikipedia.org/wiki/Projet:Wiki-Day [consulté le 6 avril 2020].

5 Pour un retour sur ces différents projets, on pourra consulter la page Wikipédia de ces projets pédagogiques

https://fr.wikipedia.org/wiki/Wikipédia:Projets_pédagogiques/Master_Patrimoine_et_Musées/Université_Bordeaux_Montaignt ainsi que : Jessica de Bideran, "L'atelier des médiations ou Wikipédia au musée », en ligne sur Com'en Histoire, 12 septembre 2016 : https://cehistoire.hypotheses.org/69o [consultés le 6 avril 2020].

6 Service du patrimoine documentaire - Service de coopération documentaire du réseau des bibliothèques des universités de Bordeaux : https://bibliotheques.u-bordeaux.fr/Lesbibliotheques/Patrimoine-documentaire [consulté le 6 avril 2020].

7 Nous reprenons ici l'expression formulée par Lionel Barbe à la suite de ses propres expériences : https://www.wikimedia.fr/du-puits-de-science-au-passeur-de-science-enseigner-avec-wikipedia/ [consulté le 6 avril 2020].

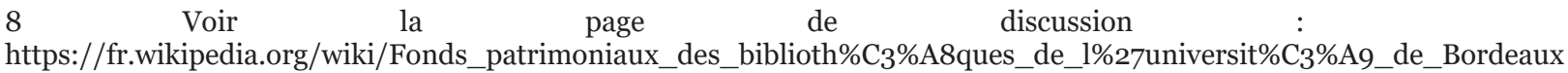
[consulté le 6 avril 2020].

9 Dont les pages utilisateurs sont les suivantes : https://fr.wikipedia.org/wiki/Discussion_utilisateur:William_Ellison et https://fr.wikisource.org/wiki/Utilisateur:C\%C3\%A9C\%C3\%Ā9dille [consultées le 6 avril 2020].

10 Comme le précise la page dédiée sur Wikipédia : « Être patrouilleur ne consiste pas à avoir un statut mais à effectuer bénévolement une maintenance de l'encyclopédie » (https://fr.wikipedia.org/wiki/Wikip\%C3\%A9dia:Patrouille_RC [consulté le 6 avril 2020]).

11 MediaWiki est le logiciel qui est utilisé pour l'édition des pages de Wikipédia, avec un cadre technique très précis pour la mise en forme des contributions proposées par les internautes (https://www.mediawiki.org/wiki/MediaWiki [consulté le 6 avril 2020]).

12 Pour connaitre l'ensemble de ces principes fondateurs, voir : https://fr.wikipedia.org/wiki/Wikip\%C3\%A9dia:Principes_fondateurs [consulté le 6 avril 2020]). 
13 C'est-à-dire : (1) le patrimoine de l'Université de Bordeaux en veillant à se limiter aux informations de niveau encyclopédique ; (2) les grands personnages de l'université susceptibles d'être admissibles au sein d'une encyclopédie généraliste (Gaston Lespiault, Edmond-Gustave Camus, Philippe Tissié, Jules Hoüel) ; (3) et l'histoire du livre et de la bibliophilie avec comme perspective l'alimentation d'articles transversaux en les complétant par l'ajout de ressources rares (rubrique typographique, incipits, frontispices, incunables, volvelles).

14 Citons la réaction du Musée d'ethnographie de l’Université de Bordeaux qui a fait l'objet d'une création d'article dans le cadre de ce projet, ces fonds patrimoniaux relevant des compétences de la bibliothèque universitaire, et qu'il a fallu convaincre de collaborer avec les étudiants malgré leur méfiance initiale.

15 http://www.babordnum.fr/ [consulté le 6 avril 2020].

16 Voir : https://www.inha.fr/fr/recherche/le-departement-des-etudes-et-de-la-recherche/domainesde-recherche/programmes-en-cours/images-usages.html [consulté le 6 avril 2020].

\title{
Pour citer cet article
}

Référence papier

Jessica de Bideran et Romain Wenz, «Contribuer à la diffusion du patrimoine documentaire sur Wikipédia : pratiques et enjeux pour les institutions culturelles », Culture \& Musées, 35 | 2020, 165188.

Référence électronique

Jessica de Bideran et Romain Wenz, «Contribuer à la diffusion du patrimoine documentaire sur Wikipédia : pratiques et enjeux pour les institutions culturelles », Culture \& Musées [En ligne], 35 | 2020, mis en ligne le 01 juin 2020, consulté le 31 mai 2021. URL :

http://journals.openedition.org/culturemusees/4762 ; DOI : https://doi.org/10.4000/culturemusees.4762

\begin{abstract}
Auteurs
Jessica de Bideran

Université Bordeaux Montaigne, MICA

Actuellement ingénieure de recherche et chargée d'enseignement à l'Université Bordeaux Montaigne, Jessica de Bideran est en charge de plusieurs programmes de recherche qui interrogent, par la mise en réseau de professionnels et la mise en pratique de différents programmes de numérisation et de valorisation, l'impact du numérique sur la diffusion et la réception des objets patrimoniaux. Après une thèse consacrée aux restitutions infographiques de sites archéologiques et monuments historiques, ses recherches en sciences de l'information et de la communication se consacrent à l'étude des pratiques numériques au sein des institutions culturelles (musées, archives, bibliothèques, etc.) comme au processus de médiation numérique (usages et circulation numériques des ressources patrimoniales). En 2019, elle a publié : « Numérisation et extension du patrimoine littéraire », in Fabienne Henryot (dir), La Fabrique du patrimoine écrit ; « Quand le numérique fait musée », Revue française des sciences de l'information et de la communication. En 2017, elle est l'auteure de : "Quand les experts du patrimoine s'emparent du transmédia storytelling. L'exemple de Montaigne Superstar, une stratégie inachevée », Communication \& Organisation ; "Restituer le Moyen Âge et ses monuments en images de synthèse, le palais de l'Ombrière comme objet médiateur ", in Daniel Jacobi et Fabrice Denise (dir.), Les Médiations de l'archéologie.

jessica.de-bideran[at]u-bordeaux-montaigne.fr
\end{abstract}

\section{Romain Wenz}

Université de Bordeaux

Responsable du service du patrimoine documentaire (Service de coopération - Université de Bordeaux) depuis 2017, Romain Wenz encadre l'équipe chargée du patrimoine : atelier de reliure et de numérisation, conservation partagée et bibliothèque numérique. De 2009 à 2013, il a travaillé comme conservateur à la Bibliothèque nationale de France où il était chef de projet métier pour data.bnf.fr, puis, de 2015 à 2017, au service des Archives de France où il a mis en place le portail francearchives.fr. Archiviste-paléographe de formation, il est l'auteur d'une thèse de l'École des chartes sur la législation du port d'armes à la fin du Moyen Âge, d'un mémoire Enssib (diplôme de conservateur) et de plusieurs publications sur les traitements de données issues des catalogues de bibliothèques. II est l'auteur de "L'open data, un levier pour l'évolution des catalogues », publié dans l'ouvrage Vers de nouveaux catalogues dirigé par Emmanuelle Bermès en 2016, et « Des catalogues aux métadonnées : la bibliothèque vers le web sémantique " dans Bibliothèques en France, 19982013 sous la direction d'Yves Alix en 2013.

Courriel : romain.wenz[at]u-bordeaux.fr

\section{Droits d'auteur}

Culture \& Musées 